\title{
Realize molecular surgical knife in tumor therapy by nanotechnology
}

\section{Yadong Li}

In cancer therapy, the main challenge is how to attack the tumor and avoid injuring any normal organs in the meantime. In the last decades, scientists have made great efforts to try targeting the tumors, but little progress is achieved because all of the known therapeutic techniques could not latch the tumor cells down and always bring tremendous harms to normal cells or organs. The recent research by Professor Chunru Wang and his colleagues from Institute of Chemistry, Chinese Academy of Sciences reported a novel strategy for tumor therapy that aims at the tumor blood vessels and can precisely destroy them, shedding light on tumor-targeting therapy by the advanced nanotechnology.

Different from most researches targeting at the cancer cells, Wang et al. noticed the structural defects in tumor blood vessels, where wide spread of nano-gaps between endothelia cells lead to a hyperpermeable feature of them for nanoparticles up to hundreds of nanometers. In fact, the typical defective structure of tumor blood vessels has been widely used to deliver and enrich nanomaterials to tumors, which is well known as enhanced permeation and retention (EPR) effect. Now in Wang's paper, these typical tumor vascular defects provide a new use to specifically destroy the tumor blood vessels (Fig. 1), which cuts off the nutrition supply of tumor tissues and leads to a rapid tumor necrosis in only $0.5-1$ hour.

Wang's main interests are in fact on fullerenes and metallofullerenes, so he knows well the amazing explosive phase transition effect of metallofullerene Gd@C $\mathrm{C}_{82}$ crystalline structures while being heated up or simply irradiated by radiofrequency (RF). They synthesized water-soluble Gd@ $\mathrm{C}_{82}$ nanocrystals (GFNCs) and intravenously injected the solution to tumor-bearing mice, and following blood circulation, these $\mathrm{Gd} @ \mathrm{C}_{82}$ nanoparticles reached the tumor and tried to permeate the blood vessels. By this time, an RF

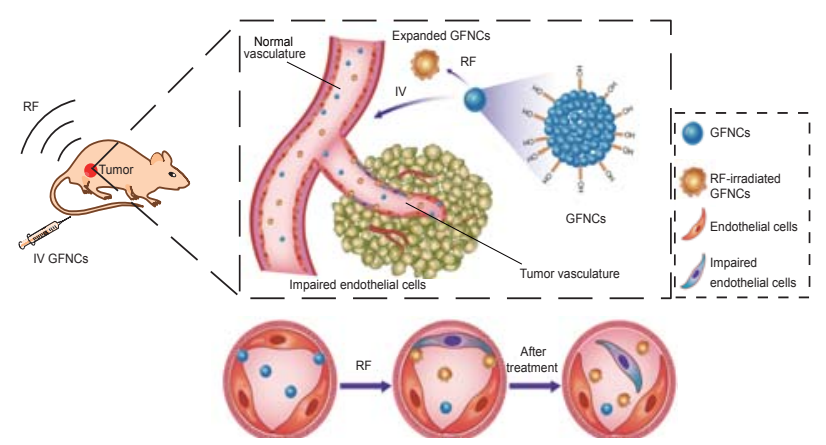

Figure 1 A schematic draw of the antitumor mechanism of RF-assistant GFNCs treatments. The GFNCs are first accumulated in the lining of the tumor blood vessels due to EPR effects, and then the sudden volume expansion and the rotation of the RF-assistant GFNCs induce the exfoliation of endothelia cells.

irradiation was applied and the vascular embedded Gd@ $\mathrm{C}_{82}$ nanocrystals would undergo significant size-expanding through a phase transition (from ca. 130 to ca. $200 \mathrm{~nm}$ ), which is observed to effectively exfoliate the surrounding endothelia cells, resulting in rapid tumor necrosis and shrinkage.

This tumor therapeutic strategy perfectly combines biology and nanotechnology, which precisely targets the harms on the defective tumor blood vessels, and no hurts are observed to normal tissues. Because any solid tumors must have blood vessels, this strategy is expected to use on various tumors such as liver cancer, breast cancer, lung cancer, etc.

Received October 11 2015; accepted October 12 2015; published online 26 October 2015

1 Zhen M, Shu C, Li J, et al. A highly efficient and tumor vascular-targeting therapeutic technique with size-expansible gadofullerene nanocrystals. Sci China Mater, 2015, 58: 799-810 